\title{
HLA-E expression in diffuse glioma: relationship with clinicopathological features and patient survival
}

\author{
Zhifeng $\mathrm{Wu}^{1 \dagger}$, Jingshan Liang ${ }^{1,2+}$, Zheng Wang ${ }^{3}$, Aimin $\mathrm{Li}^{2}$, Xing Fan ${ }^{1 *}$ (D) and Tao Jiang ${ }^{1,3^{*}}$
}

\begin{abstract}
Background: Human leukocyte antigen-E (HLA-E) has been extensively investigated in various human cancers including glioma. However, the clinical significance of HLA-E expression in glioma patients has not been elucidated. The current study aimed to investigate the association of HLA-E expression with clinicopathological features and survival in patients with diffuse glioma.

Methods: A total of 261 glioma patients were enrolled, subsequently, mRNA microarray analysis was conducted to identify the relationship of HLA-E with clinicopathological features and patient survival.

Results: HLA-E was significantly overexpressed in high-grade gliomas compared to low-grade gliomas (LGGs). Moreover, HLA-E expression was significantly higher in diffuse astrocytomas than oligodendrogliomas $(p=0.032, \mathrm{t}-$ test). Kaplan-Meier analysis showed that progression-free survival (PFS) and overall survival (OS) were significantly better in LGG patients with low HLA-E expression ( $p=0.018$ for PFS and $p=0.020$ for OS, Log-rank test).

Furthermore, HLA-E expression was identified to be an independent prognostic factor by Cox analysis ( $p=0.020$ for PFS and $p=0.024$ for OS).

Conclusions: This is the first study which identified the clinical significance of HLA-E in diffuse glioma. HLA-E expression was correlated with more aggressive tumor grade and histological type and was identified as an independent prognostic biomarker in LGG patients.
\end{abstract}

Keywords: Diffuse glioma, HLA-E, Clinical outcome, Prognostic biomarker

\section{Background}

According to the 2016 World Health Organization (WHO) classification [1], the diffuse gliomas include the WHO grade II/III astrocytic tumors, the grade II/III oligodendrogliomas and the grade IV glioblastomas (GBM). They are the most frequent intracranial tumors in adults, and account for over $80 \%$ of primary brain neoplasms [2]. To date, the prognosis of patients with diffuse gliomas remains disappointing despite conventional therapies (maximal tumor resection followed by radiotherapy and concomitant/adjuvant temozolomide) [3]. In the coming era of "precision medicine", a more precise, individualized treatment strategy based on

\footnotetext{
*Correspondence: xingkongyaoxiang@163.com; taojiang1964@163.com ${ }^{\dagger}$ Zhifeng Wu and Jingshan Liang contributed equally to this work.

${ }^{1}$ Beijing Neurosurgical Institute, Capital Medical University, Beijing, China Full list of author information is available at the end of the article
}

genetic, biomarker, phenotypic, or psychosocial characteristics would be more advocatable for patients with diffuse gliomas.

"Tumor-promoting inflammation" and "avoiding immune destruction" are considered as two of the hallmarks of cancer [4]. It has been widely accepted that glioma can suppress antitumor immune responses, and the underlying mechanisms are thought to be quite complex $[5,6]$. A better understanding on the mechanisms of glioma-mediated immune suppression can help us to develop more selective and effective cancer treatments, i.e. immunotherapies. So far, remarkable progress has been achieved in this field and various types of immunotherapies, such as adoptive $\mathrm{T}$-cell therapies and immune checkpoint therapies, have been considered as promising new modalities for glioma treatment [7-9].

(c) The Author(s). 2020 Open Access This article is distributed under the terms of the Creative Commons Attribution 4.0 International License (http://creativecommons.org/licenses/by/4.0/), which permits unrestricted use, distribution, and 
However, the mechanisms behind glioma-mediated immune suppression are still far from clear.

The major histocompatibility complex (MHC) comprises a set of genes that are essential to the immune response modulation. The human $\mathrm{MHC}$, i.e. human leukocyte antigen (HLA) complex, is a 4000-kb gene complex on chromosome 6 region $6 \mathrm{p} 21.31$. It includes over 50 recognized genes and can be separated into three subgroups: HLA-I, HLA-II and HLA-III [10]. HLA molecules are well-known for their major roles in the modulation of human immune system, and aberrant expression of HLA molecules has been indicated in multiple types of human cancers, such as breast cancer, prostate cancer and non-small cell lung cancer [11-13]. The association between HLA and glioma was investigated early in 1978, the authors determined HLA-typing of 80 glioma patients and compared the antigen frequencies with normal controls, but no significant results were found [14]. However, in recent decades, some significant findings on the relationship of HLA with glioma have been reported. For instance, Diao et al. identified a negative correlation of HLA-DR expression to patient survival in glioma patients [15].

HLA-E and HLA-G are both representative molecules of MHC class Ib (nonclassical), which differs from MHC class Ia (classical) by its nonpolymorphic and conserved nature [16]. Our group once investigated the clinical significance of HLA-G expression in glioma patients and identified that HLA-G expression was a potential biomarker for predicting aggressive entities of glioma, moreover, it could also serve as an independent predictor of poor clinical outcomes in patients with lowgrade gliomas (LGG, WHO grade II) [17, 18]. Accordingly, we hypothesized that HLA-E might be also a potential biomarker for glioma patients to predict aggressive entities and poor clinical outcomes. To the best of our knowledge, there have been only one clinicalpathological correlative study which investigated the clinical significance of HLA-E expression in gliomas [19]. However, the research objectives were only limited to GBM patients, and the sample size was too small (39 cases). In the current study, we performed a large sample analysis to investigate the clinicopathological significance of HLA-E in human diffuse gliomas.

\section{Methods}

\section{Patients and tissue samples}

The data from 305 consecutive patients treated for diffuse glioma at the Glioma Center of Beijing Tiantan Hospital between June 2007 and September 2013 were retrospectively reviewed. After excluding 44 patients with secondary/recurrent tumors, 261 patients with histologically confirmed primary diffuse gliomas were eventually enrolled in the study. Subsequently, the pathological diagnosis of each enrolled patient was reevaluated by an experienced neuropathologist according to the 2016 WHO classification, data of isocitrate dehydrogenase (IDH) mutation status (detected by pyrosequencing, 233 patients available) and 1p19q co-deletion status (detected by fluorescence, 236 patients available) were reviewed. Additionally, negative control brain tissue samples were collected from 5 patients with craniocerebral trauma. Clinical information (age, gender, etc.) of all patients were collected from the Chinese Glioma Genome Atlas database. The tissue samples were snap frozen and stored in liquid nitrogen immediately after resection until further processing. For reducing the influence of contamination, only samples with tumor cells over $80 \%$ would be selected for further analysis. The current study was approved by the Ethics Committee of Beijing Tiantan Hospital, and written informed consents were obtained from all patients.

\section{RNA extraction and whole genome mRNA expression profiling}

RNA isolation and microarray analysis were performed as previously described [18]. Briefly, first, total RNA was isolated by MirVana miRNA Isolation kit (Thermo Fisher Scientific, Waltham, USA). Then a NanoDrop ND-1000 spectrophotometer (NanoDrop Technologies, Wilmington, USA) was subsequently used to perform the quantification of exacted RNA. Total RNA integrity was checked by an Agilent 2100 Bioanalyzer (Agilent, California, USA), Messenger RNA expression microarray was performed on all samples with the Agilent Whole Human Genome Array (Agilent, California, USA). Data acquisition was carried out by the Agilent G2565BA Microarray Scanner System and Agilent Feature Extraction Software (version 9.1). The normalization of probe intensities was performed using GeneSpring GX 11.0. Normalized gene expression value were log-transformed for analysis.

\section{Statistical analysis}

All statistical analyses were performed with the $\mathrm{R}$ project for statistical computing (www.r-project.org) and GraphPad Prism (version 8.0.1 for Windows, GraphPad Software, San Diego, California, USA). Variables were compared using Chi-square test, Student t-test, MannWhitney $\mathrm{U}$ test, or Fisher exact test as appropriate, and a $p$-value $<0.05$ was considered statistically significant. Continuous variables were expressed by mean and standard deviation (SD). For survival analysis, patients were divided into two subgroups based on their HLA-E mRNA expression values (cut off at 50\% of the group). Kaplan-Meier method (Log-rank test) was performed to investigate the correlation of HLA-E mRNA expression levels with progression-free survival (PFS) and overall 
survival (OS) in each tumor grade. PFS was defined as the time from surgery until tumor recurrence (identified by neuroimaging according to the McDonald or the Response Assessment in Neuro-Oncology criteria), while OS was defined as the time from surgery until death or date of last follow-up. At last, Cox regression analysis was used to further evaluate the prognostic value of the identified markers.

\section{Results}

\section{Clinical characteristics}

Clinical information and mRNA expression microarray data of 261 patients were obtained. Among these patients, 117 were diagnosed as LGGs (62 diffuse astrocytomas, IDH-mutant, 22 diffuse astrocytomas, IDH-wildtype; 7 diffuse astrocytomas, NOS; 12 oligodendrogliomas, IDHmutant and 1p19q-codeleted; 14 oligodendrogliomas, NOS), 36 as anaplastic gliomas (AG, WHO Grade III; 13 anaplastic astrocytomas, IDH-mutant; 14 anaplastic astrocytomas, IDH-wildtype; 3 anaplastic oligodendrogliomas, IDH-mutant and 1p19q-codeleted; 5 anaplastic oligodendrogliomas, NOS; 1 anaplastic oligoastrocytoma, NOS), 108 as GBMs (12 GBMs, IDH-mutant; 81 GBMs, IDH-wildtype; 15 GBMs, NOS). The clinical characteristics of all patients are shown in Table 1.

\section{HLA-E mRNA expression in diffuse gliomas}

HLA-E expression in normal brain tissues and diffuse glioma tissues of each grade are shown in Fig. 1. A significant positive correlation of HLA-E expression and tumor grade was identified, HLA-E gene was highly overexpressed in GBMs $(0.286 \pm 0.787)$, followed by AGs $(0.176 \pm 0.824)$ and LGGs $(-0.340 \pm 0.802)$. Even in LGGs, the expression of HLA-E was significantly higher than it in normal brain tissues $(-0.340 \pm 0.802$ versus $0.863 \pm 0.228, \quad p=0.002, \quad$ t-test, Fig. 1). Significant

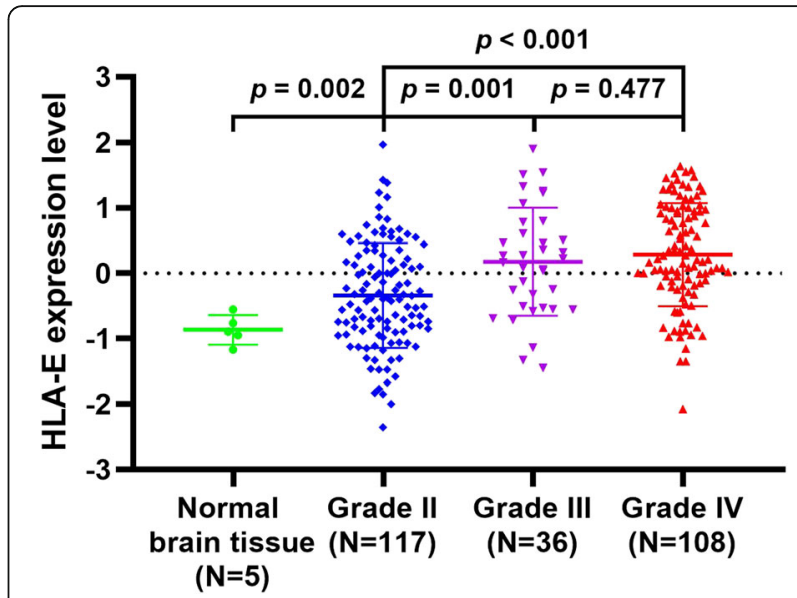

Fig. 1 HLA-E expression levels in normal samples $(N=5)$ and different grades of gliomas $(N=261)$

differences in HLA-E expression could be observed between low-grade and high-grade glioma (HGG, Grade III and IV) tissues ( $p=0.001$ and $p<0.001$, t-test, LGGs versus AGs and LGGs versus GBMs, respectively, Fig. 1), while no significant difference in HLA-E expression was identified between AGs and GBMs $(p=0.477$, t-test, Fig. 1). Expression levels of HLA-E in 5 normal brain tissues are summarized in Supplementary Table S1.

To further identify the clinical significance of HLA-E expression in diffuse glioma, we also compared HLA-E mRNA expression between astrocytic tumors and oligodendroglial tumors in LGGs and AGs. We found that HLA-E expression was significantly higher in diffuse astrocytomas than it in oligodendrogliomas $(-0.255 \pm$ 0.762 versus $-0.636 \pm 0.879, p=0.032$, t-test, Fig. 2a), while no such difference was observed between anaplastic astrocytomas and anaplastic oligodendrogliomas ( $p=$ 0.671, t-test, Fig. 2b).

Table 1 Clinical characteristics of enrolled patients with diffuse gliomas ( $N=261)$

\begin{tabular}{|c|c|c|c|}
\hline Characteristics & Grade II $(N=117)$ & Grade III $(N=36)$ & Grade IV $(N=108)$ \\
\hline Median age (range, yrs) & $\begin{array}{l}38 \\
(18-61)\end{array}$ & $\begin{array}{l}42.5 \\
(18-66)\end{array}$ & $\begin{array}{l}50.5 \\
(13-70)\end{array}$ \\
\hline Gender (male) & 69 & 21 & 66 \\
\hline Side (Left) & 58 & 17 & 58 \\
\hline KPS $>80$ & 70 & 16 & 33 \\
\hline \multicolumn{4}{|l|}{ Pathology } \\
\hline Diffuse astrocytoma/GBM, IDH mutant & 62 & 13 & 12 \\
\hline Diffuse astrocytoma/GBM, IDH wildtype & 22 & 14 & 81 \\
\hline Diffuse astrocytoma/GBM, NOS & 7 & & 15 \\
\hline Oligodendroglioma, IDH mutant and 1p19q co-deleted & 12 & 3 & \\
\hline Oligodendroglioma, NOS & 14 & 5 & \\
\hline Oligoastrocytoma, NOS & & 1 & \\
\hline
\end{tabular}

KPS Karnofsky performance score, IDH isocitrate dehydrogenase 


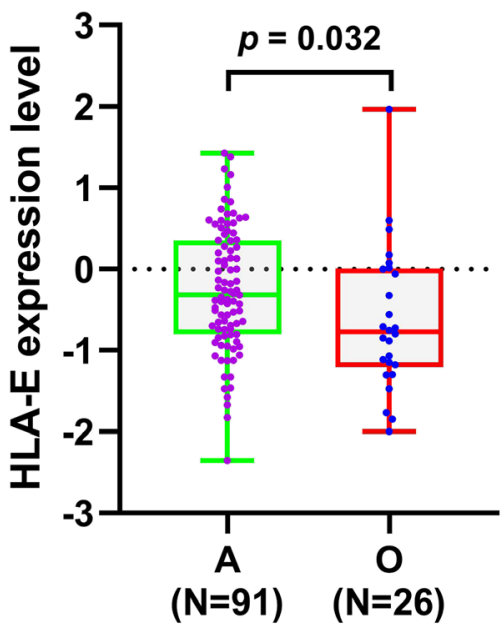

A

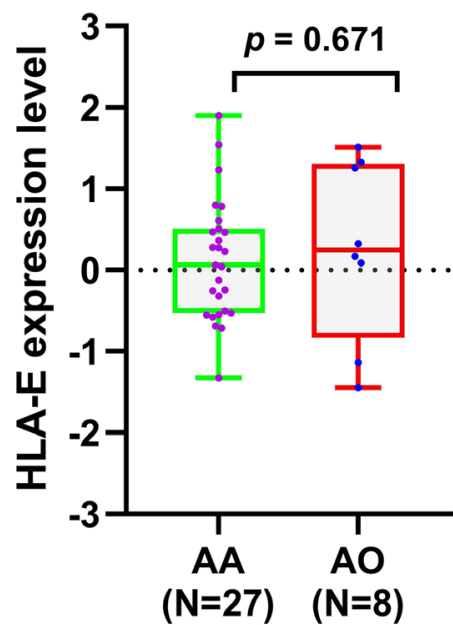

B

Fig. 2 Comparison of HLA-E mRNA expression between astrocytic tumors and oligodendroglial tumors in low-grade gliomas and anaplastic gliomasA, astrocytoma; O, oligodendroglioma; AA, anaplastic astrocytoma; AO, anaplastic oligodendroglioma.

Additionally, we also compared HLA-E mRNA expression levels according to IDH1 mutation status in diffuse gliomas of each grade, no association between HLA-E expression and IDH1 mutation status was identified (Grade II, $p=0.830$; Grade III, $p=0.400$; Grade IV, $p=$ 0.412 , t-test, Fig. S1).

\section{Survival analysis}

The follow-up rate was $96.6 \%$ with 9 patients lost follow-up. The mean follow-up time of the 252 patients was 54.9 months (range $0.9-138.1$ months). Kaplan-
Meier analysis was performed to investigate the prognostic value of HLA-E mRNA expression. For patients with LGGs, those with low HLA-E expression tended to have significantly longer PFS $(p=0.018$, Log-rank test, Fig. 3a) and OS ( $p=0.020$, Log-rank test, Fig. 3b). In order to further investigate the predictive value of HLAE expression in LGG patients, we performed KaplanMeier analysis in patients with diffuse astrocytomas and oligodendrogliomas respectively. For patients with diffuse astrocytomas, lower HLA-E expression still seemed to correlate with better clinical outcomes, but the results

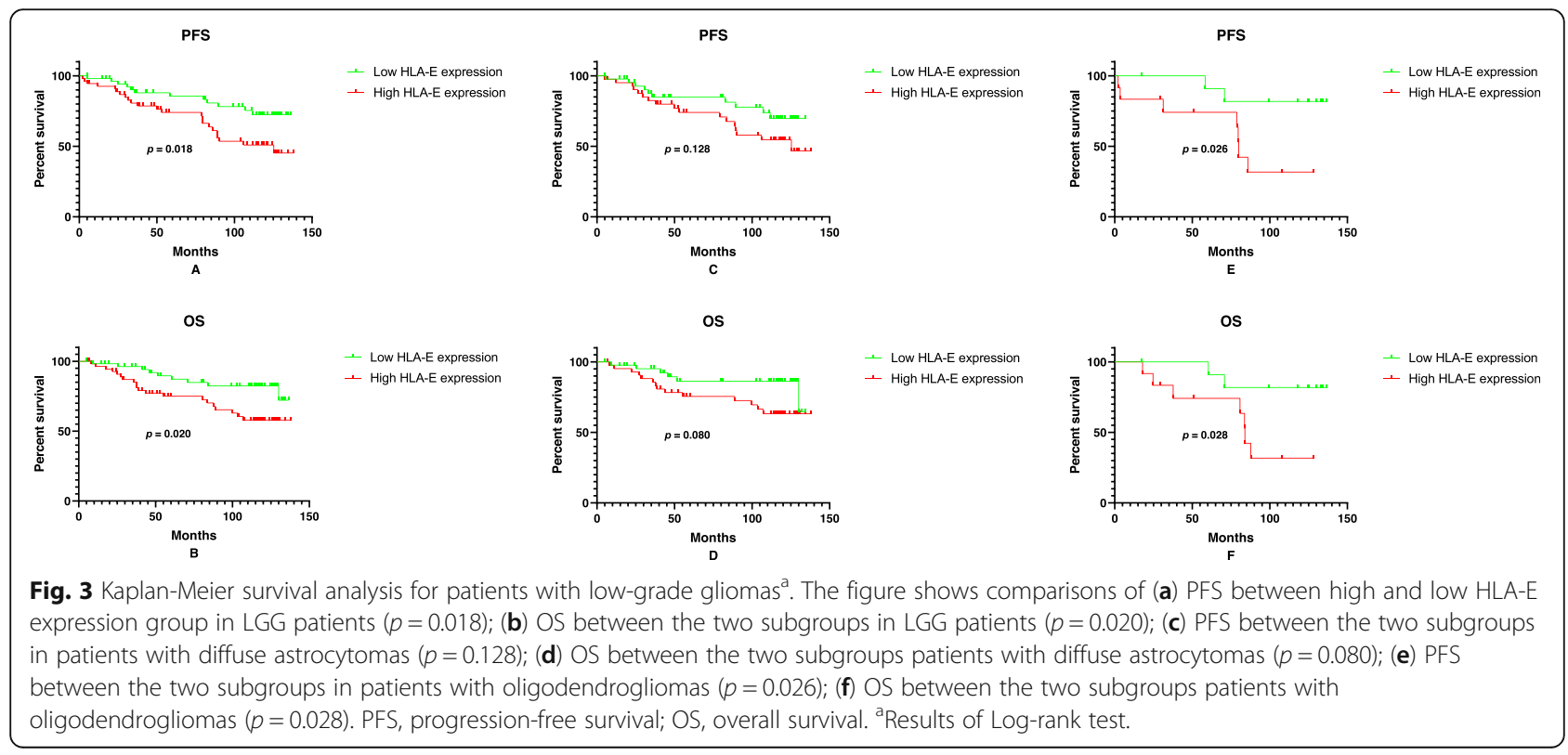


were not statistically significant ( $p=0.128$ for PFS and $p=0.080$ for OS, Log-rank test, Fig. 3c and d). As for patients with oligodendrogliomas, low HLA-E expression remained to be associated with improved PFS $(p=0.026$, Log-rank test, Fig. 3e) and OS ( $p=0.028$, Log-rank test, Fig. 3f). In contrast, no significant differences were observed with respect to either PFS or OS between patients with low and high HLA-E expression in patients with AGs or GBMs (Fig. S2).

\section{Cox regression analysis}

As the previous Kaplan-Meier analyses identified that HLA-E mRNA expression served as a prognostic factor for patients with LGGs rather than patients with AGs or GBMs, Cox regression analysis was performed only in patients with LGGs, and HLA-E expression was enrolled as a continuous variable. HLA-E expression was identified as an independent predictor for poor clinical outcomes $(p=0.020$ and 0.024 , for PFS and OS, respectively, Table 2). Meanwhile, Karnofsky performance score $(p<0.001$ both for PFS and OS, Table 2) and age at diagnosis $(p=0.002$ and 0.005 , for PFS and OS, respectively, Table 2) were also identified as independent predictive factors for the clinical outcomes of LGG patients.

\section{Discussion}

Glioma is considered as a disease accompanied by profound genomic alterations. In recent decades, great progress has been made in understanding the molecular pathological basis of glioma. The identification of tumor-specific genetic alterations, such as IDH mutation, $1 \mathrm{p} 19 \mathrm{q}$ co-deletion, telomerase reverse transcriptase promoter mutation, etc. has refreshed our understanding of this lethal disease [20,21]. However, the understanding about the molecular information of glioma remains insufficient. For instance, the role of immune-related molecular changes in glioma has not been fully elucidated.

HLA-E is one of the most extensively investigated MHC class Ib molecules and plays a double role in both innate and adaptive immunity. Aberrant expression of HLA-E has been identified in various human malignancies, such as breast cancer, gastric cancer, rectal cancer, and colorectal cancer [22-26]. As for glioma, HLA-E has been reported to be elevated in human glioblastomas by immunohistochemistry, which was in consistent with what we demonstrated by microarray analysis in this study [19]. Additionally, previous studies also explored the role HLA-E played in the occurrence and development of glioma. It has been identified that HLA-E up-regulation in glioma cells could result in enhanced resistance to NK cell-mediated immune response [27], moreover, the interaction of HLA-E on glioma cells with CD94/NKG2A (a human NK cell inhibitory receptor on lymphocytes) could also compromise innate antitumor immune responses [28].

In the current study, we systematically evaluated HLAE mRNA expression in adult diffuse gliomas and found that HLA-E mRNA expression in diffuse glioma tissues was significantly higher than that in normal brain tissues, additionally, the expression level of HLA-E increased as the tumor grade progressed. Such result suggested that glioma cells had strong ability in immune resistance, which increased with the elevation of tumor grade. Additionally, our analysis also identified that the expression pattern of HLA-E was also associated with the origin of tumor cells. Compared with oligodendrogliomas, diffuse astrocytomas showed higher expression of HLA-E. It showed that diffuse astrocytomas had stronger ability in immune resistance and therefore more aggressive than oligodendrogliomas, which was in conformity to our clinical knowledge.

The association between HLA-E expression and IDH1 mutation was also investigated. IDH1 mutation has been identified to be one of most important molecular pathological alterations in glioma, but HLA-E showed little association with it. This suggested that the HLA-E driven progression might be independent of IDH status in diffuse glioma.

The prognostic value of HLA-E has been verified in various of cancers. For instance, expression of HLA-E has been reported to resulted in a worse relapse-free period for patients with breast cancer [22]. Another study demonstrated that overexpression of HLA-E was

Table 2 Predictors of progression-free survival and overall survival on multivariate analysis in patients with low-grade gliomas ${ }^{a}$

\begin{tabular}{|c|c|c|c|c|c|c|}
\hline \multirow[t]{2}{*}{ Parameters } & \multicolumn{3}{|l|}{ PFS } & \multicolumn{3}{|l|}{ OS } \\
\hline & Risk ratio & $95 \% \mathrm{Cl}$ & $p$-value & Risk ratio & $95 \% \mathrm{Cl}$ & $p$-value \\
\hline HLA-E expression & 1.717 & $1.088-2.710$ & 0.020 & 1.778 & $1.080-2.926$ & 0.024 \\
\hline Age & 1.065 & $1.023-1.109$ & 0.002 & 1.072 & $1.022-1.125$ & 0.005 \\
\hline Sex (male) & 0.909 & $0.452-1.839$ & 0.790 & 1.484 & $0.681-3.233$ & 0.321 \\
\hline KPS & 0.899 & $0.862-0.938$ & $<0.001$ & 0.906 & $0.866-0.947$ & $<0.001$ \\
\hline Side (Left) & 0.899 & $0.452-1.788$ & 0.761 & 1.317 & $0.608-2.853$ & 0.486 \\
\hline
\end{tabular}

PFS Progression-free survival, OS overall survival, LGG low-grade glioma, Cl confidence interval, KPS Karnofsky performance score

${ }^{\text {a }}$ Results of Cox regression analysis 
related to a lower five-year survival rate in patients with gastric cancer [25]. Consistent with these findings in other cancers, we also identified a correlation of lower HLA-E mRNA expression with better PFS and OS in LGG patients. Furthermore, HLA-E expression was identified as an independent prognostic factor of clinical outcomes for LGG patients by Cox analysis. As for HGG, Kren et al. once reported an unexpected positive correlation of HLA-E expression to patient survival in 39 cases of GBM [19]. However, here in 36 cases of AG and 108 cases of GBM, no associations between HLA-E expression and patient survival were identified. One potential explanation is that the interaction between the immune system and tumor was relatively slow, and HGG progresses so rapidly that the immune system has no enough time to exert its influence on tumors. Moreover, a previous study showed that the cellular cytotoxicity mediated by Cetuximab, an anti-epidermal growth factor receptor monoclonal antibody, could be inhibited by HLA-E membrane expression in colon cancer cells [29]. Accordingly, immunotherapy may show better efficacy on LGG patients with low HLA-E expression, especially for those with oligodendrogliomas.

The current study had its limitations. First, all the HLA-E expression data are from microarray with no experimental verification. Moreover, data are exclusively from CGGA, which may add the systematic bias. Further studies are needed to verify the results, and experimental studies should be performed to investigate the role of HLAs in the development of glioma.

\section{Conclusions}

In the current study, by analyzing mRNA expression microarray data of 261 patients with diffuse gliomas, the correlations of HLA-E expression with tumor grade and histological type in diffuse glioma were identified. Moreover, HLA-E expression was found to be negatively related to clinical outcomes and could serve as an independent prognostic factor in patients with LGGs. Further investigation into the roles of HLA-E and other HLA molecules in glioma can help us to obtain a better understanding of the interaction between glioma and the immune system and promote the development of relevant immunotherapy.

\section{Supplementary information}

Supplementary information accompanies this paper at https://doi.org/10. 1186/s12883-020-01640-4.

Additional file 1: Table S1. HLA-E expression levels in negative controls (5 normal brain samples). Figure S1. HLA-E mRNA expression levels according to IDH1 mutation status in diffuse gliomas of each grade. Figure S2. Kaplan-Meier survival curves. No correlations were identified between HLA-E expression and (A) PFS; (B) OS in patients with AGs, while no correlations were identified between HLA-E expression and (C) PFS; (D) OS in patients with GBMs ${ }^{\mathrm{a}}$.

\section{Abbreviations}

AG: Anaplastic glioma; GBM: Glioblastoma; HLA: Human leukocyte antigen; IDH: Isocitrate dehydrogenase; LGG: Low-grade glioma; MHC: Major histocompatibility complex; OS: Overall survival; PFS: Progression-free survival; SD: Standard deviation; WHO: World Health Organization

\section{Acknowledgements}

We would like to thank Yuling Yang for tissue sample collection and clinical data retrieval.

\section{Authors' contributions}

ZFW: data analysis and interpretation, drafting of the manuscript; JSL: acquisition of Data, drafting of the manuscript; ZW: data analysis and interpretation; AML: critical revision of the manuscript; XF: study concept and design, critical revision of the manuscript; TJ: study concept and design, study supervision. All authors read and approved the final manuscript.

\section{Funding}

This work was supported by funding from The National Key Research and Development Plan (2016YFC0902500) and National High Technology Research and Development Program (2015CB755500). The funding was used to support the collection, analysis, and interpretation of data.

\section{Availability of data and materials}

The dataset analyzed during the current study is available in the Chinese Glioma Genome Atlas database (http://www.cgga.org.cn/download.jsp, Part D), and the dataset ID is "mRNA-array".

\section{Ethics approval and consent to participate}

The current study was approved by the Ethics Committee of Beijing Tiantan Hospital. All procedures performed in studies involving human participants were in accordance with the ethical standards of the institutional and/or national research committee and with the 1964 Helsinki declaration and its later amendments or comparable ethical standards. Written informed consents were obtained from all individual participants included in the study.

Consent for publication

Not applicable.

\section{Competing interests}

The authors declare that they have no competing interests.

\section{Author details}

${ }^{1}$ Beijing Neurosurgical Institute, Capital Medical University, Beijing, China. ²Department of Neurosurgery, Lianyungang First People's Hospital, Xuzhou Medical University, Jiangsu, China. ${ }^{3}$ Department of Neurosurgery, Beijing Tiantan Hospital, Capital Medical University, Beijing, China.

Received: 20 March 2019 Accepted: 10 February 2020

Published online: 17 February 2020

References

1. Louis DN, Perry A, Reifenberger G, von Deimling A, Figarella-Branger D, Cavenee WK, et al. The 2016 World Health Organization classification of tumors of the central nervous system: a summary. Acta Neuropathol. 2016; 131(6):803-20.

2. Ostrom QT, Bauchet L, Davis FG, Deltour I, Fisher JL, Langer $C E$, et al. The epidemiology of glioma in adults: a "state of the science" review. Neurooncology. 2014;16(7):896-913.

3. Yang P, Wang Y, Peng $X$, You G, Zhang W, Yan W, et al. Management and survival rates in patients with glioma in China (2004-2010): a retrospective study from a single-institution. J Neuro-Oncol. 2013;113(2):259-66.

4. Hanahan D, Weinberg RA. Hallmarks of cancer: the next generation. Cell. 2011;144(5):646-74.

5. Platten M, Ochs K, Lemke D, Opitz C, Wick W. Microenvironmental clues for glioma immunotherapy. Curr Neurol Neurosci Rep. 2014;14(4):440. 
6. Platten M, Reardon DA. Concepts for immunotherapies in Gliomas. Semin Neurol. 2018;38(1):62-72.

7. Fecci PE, Ochiai H, Mitchell DA, Grossi PM, Sweeney AE, Archer GE, et al. Systemic CTLA-4 blockade ameliorates glioma-induced changes to the CD4+ T cell compartment without affecting regulatory T-cell function. Clin Cancer Res. 2007;13(7):2158-67.

8. Wainwright DA, Chang AL, Dey M, Balyasnikova IV, Kim CK, Tobias A, et al. Durable therapeutic efficacy utilizing combinatorial blockade against IDO, CTLA-4, and PD-L1 in mice with brain tumors. Clin Cancer Res. 2014;20(20) 5290-301.

9. Johnson LA, Scholler J, Ohkuri T, Kosaka A, Patel PR, McGettigan SE, et al. Rational development and characterization of humanized anti-EGFR variant III chimeric antigen receptor T cells for glioblastoma. Sci Transl Med. 2015; 7(275):275ra22.

10. Marsh SG, Albert ED, Bodmer WF, Bontrop RE, Dupont B, Erlich HA, et al. Nomenclature for factors of the HLA system, 2010. Tissue Antigens. 2010; 75(4):291-455.

11. Kaneko K, Ishigami S, Kijima Y, Funasako Y, Hirata M, Okumura H, et al. Clinical implication of HLA class I expression in breast cancer. BMC Cancer. 2011;11:454

12. Carretero FJ, Del Campo AB, Flores-Martin JF, Mendez R, Garcia-Lopez C, Cozar JM, et al. Frequent HLA class I alterations in human prostate cancer: molecular mechanisms and clinical relevance. Cancer Immunol Immunother. 2016;65(1):47-59.

13. Perea F, Bernal M, Sanchez-Palencia A, Carretero J, Torres C, Bayarri C, et al. The absence of HLA class I expression in non-small cell lung cancer correlates with the tumor tissue structure and the pattern of $\mathrm{T}$ cell infiltration. Int J Cancer. 2017;140(4):888-99.

14. de Moerloose P, Jeannet M, Martin-Achard A, de Tribolet N, Seiler R, Guanella N. HLA and glioma. Tissue Antigens. 1978;12(2):146-8.

15. Diao J, Xia T, Zhao H, Liu J, Li B, Zhang Z. Overexpression of HLA-DR is associated with prognosis of glioma patients. Int J Clin Exp Pathol. 2015;8(5): 5485-90.

16. Rodgers JR, Cook RG. MHC class Ib molecules bridge innate and acquired immunity. Nat Rev Immunol. 2005;5(6):459-71.

17. Wang Y, Fan $X$, Li H, Lin Z, Bao H, Li S, et al. Tumor border sharpness correlates with HLA-G expression in low-grade gliomas. J Neuroimmunol. 2015;282:1-6

18. Fan X, Wang Y, Zhang C, Liu X, Qian Z, Jiang T. Human leukocyte antigen-G overexpression predicts poor clinical outcomes in low-grade gliomas. J Neuroimmunol. 2016;294:27-31.

19. Kren L, Slaby O, Muckova K, Lzicarova E, Sova M, Vybihal V, et al. Expression of immune-modulatory molecules HLA-G and HLA-E by tumor cells in glioblastomas: an unexpected prognostic significance? Neuropathology. 2011;31(2):129-34.

20. Yan $\mathrm{H}$, Parsons DW, Jin G, McLendon R, Rasheed BA, Yuan W, et al. IDH1 and IDH2 mutations in gliomas. N Engl J Med. 2009;360(8):765-73.

21. Eckel-Passow JE, Lachance DH, Molinaro AM, Walsh KM, Decker PA, Sicotte $\mathrm{H}$, et al. Glioma groups based on 1p/19q, IDH, and TERT promoter mutations in tumors. N Engl J Med. 2015:372(26):2499-508.

22. de Kruijf EM, Sajet A, van Nes JG, Natanov R, Putter H, Smit VT, et al. HLA-E and HLA-G expression in classical HLA class I-negative tumors is of prognostic value for clinical outcome of early breast cancer patients. J Immunol. 2010:185(12):7452-9.

23. Reimers MS, Engels CC, Putter H, Morreau H, Liefers GJ, van de Velde CJ, et al. Prognostic value of HLA class I, HLA-E, HLA-G and Tregs in rectal cancer: a retrospective cohort study. BMC Cancer. 2014;14:486.

24. Guo ZY, LV YG, Wang L, Shi SJ, Yang F, Zheng GX, et al. Predictive value of HLA-G and HLA-E in the prognosis of colorectal cancer patients. Cell Immunol. 2015;293(1):10-6.

25. Ishigami S, Arigami T, Okumura H, Uchikado Y, Kita Y, Kurahara H, et al. Human leukocyte antigen (HLA)-E and HLA-F expression in gastric cancer. Anticancer Res. 2015;35(4):2279-85.

26. Huang R, Zhang D, Li F, Xiao Z, Wu M, Shi D, et al. Loss of Fas expression and high expression of HLA-E promoting the immune escape of early colorectal cancer cells. Oncol Lett. 2017;13(5):3379-86.

27. He W, Kuang Y, Xing X, Simpson RJ, Huang H, Yang T, et al. Proteomic comparison of $3 \mathrm{D}$ and $2 \mathrm{D}$ glioma models reveals increased HLA-E expression in 3D models is associated with resistance to NK cell-mediated cytotoxicity. J Proteome Res. 2014;13(5):2272-81.
28. Wischhusen J, Friese MA, Mittelbronn M, Meyermann R, Weller M. HLA-E protects glioma cells from NKG2D-mediated immune responses in vitro: implications for immune escape in vivo. J Neuropathol Exp Neurol. 2005; 64(6):523-8.

29. Levy EM, Sycz G, Arriaga JM, Barrio MM, von Euw EM, Morales SB, et al. Cetuximab-mediated cellular cytotoxicity is inhibited by HLA-E membrane expression in colon cancer cells. Innate Immun. 2009;15(2):91-100.

\section{Publisher's Note}

Springer Nature remains neutral with regard to jurisdictional claims in published maps and institutional affiliations.

\section{Ready to submit your research? Choose BMC and benefit from:}

- fast, convenient online submission

- thorough peer review by experienced researchers in your field

- rapid publication on acceptance

- support for research data, including large and complex data types

- gold Open Access which fosters wider collaboration and increased citations

- maximum visibility for your research: over $100 \mathrm{M}$ website views per year

At BMC, research is always in progress.

Learn more biomedcentral.com/submissions 